\title{
Effects of a Renal Nordic Walking Program on Quality of Life and Fitness in renal patients: A Randomized Controlled Trial
}

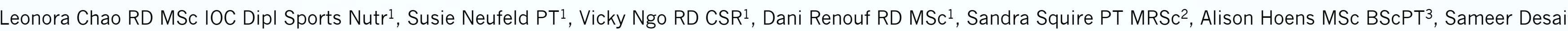
${ }^{1}$ Renal program, Providence Health Care, Vancouver, Canada, ${ }^{2}$ Professional Practice, Providence Health Care, Vancouver, Canada, ${ }^{3}$ Physiotherapy, Providence Health Care, Vancouver, Canada, ${ }^{4}$ Centre for Health Evaluation \& Outcomes Sciences (CHÉOS), Vancouver, Canada

\section{Introduction and Background}

Many persons with kidney disease do not meet physical activity guidelines. ${ }^{1}$ Based on findings that hospital-based group exercise programs can reduce fears and increase exercise self-efficacy ${ }^{2}$, and a survey supporting patient's desire for this programming, a Renal Nordic walking (NW) program at St Paul's Hospital was developed. Working with a Patient Partner throughout the phases from study design to dissemination, this study investigated the effects of a 3-month supervised Renal NW program on the fitness and quality of life of renal outpatients.

\section{Methods}

Thirty participants, aged 45.84 were randomized to NW $(n=15)$ or non-NW ( $n=15)$ groups. The NW group was offered 2 supervised NW sessions/week; the non-NW group continued their own activities. Outcome measurements at baseline and 3-month included weight, handgrip strength (HGS), 30-sec sitto-stand test, 6-min walk test (6MWT), and Kidney Disease and Quality of Life questionnaire (KDQOL-36). Daily steps were recorded using Fitbit. Median changes in outcomes from baseline to 3-months were calculated between groups.

\section{Results/Findings}

Participants included post-renal transplant $(n=10)$, predialysis $(n=14)$, hemodialysis $(n=3)$, and peritoneal dialysis $(n=3)$ patients. The NW group appeared less healthy compared to the non-NW group at baseline. However, the NW group had greater improvements in KDQOL-36 (Effect of kidney disease; $p=0.021), 6 \mathrm{MWT}$ distance $(41.5 \mathrm{~m})$, and HGS $(1.1 \mathrm{~kg})$ at $3 \cdot$ month.
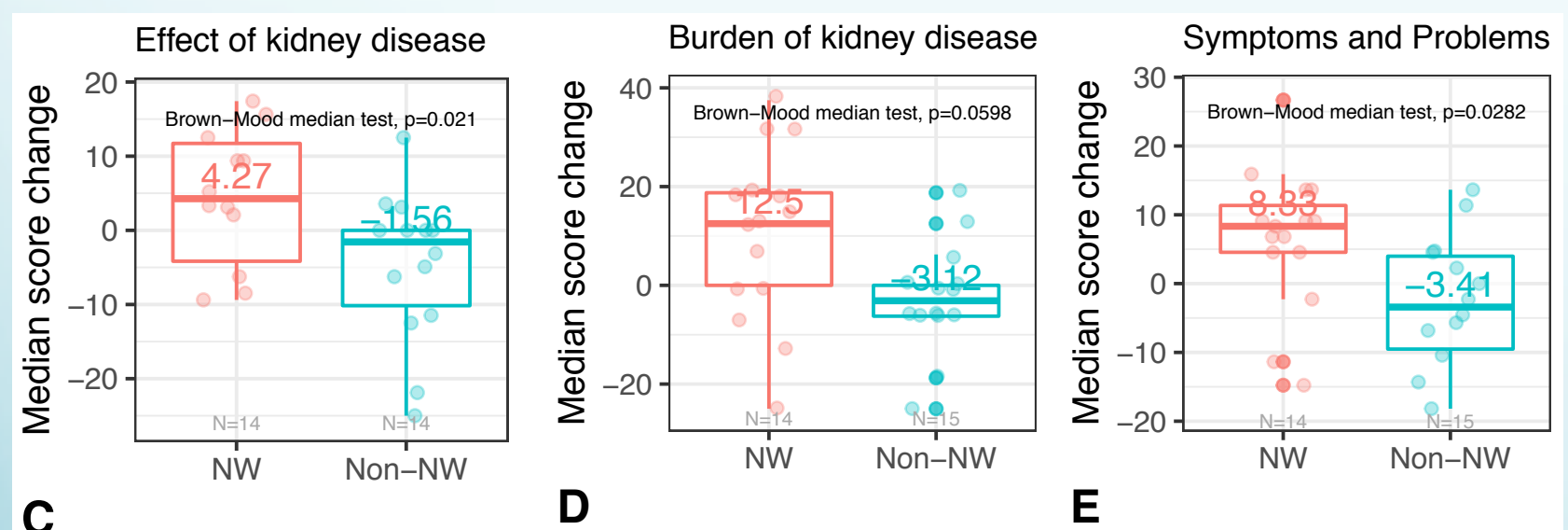

Figures C-E. Change in Quality of Life Domains of KDQOL-36 questionnaire from pre to post between groups.

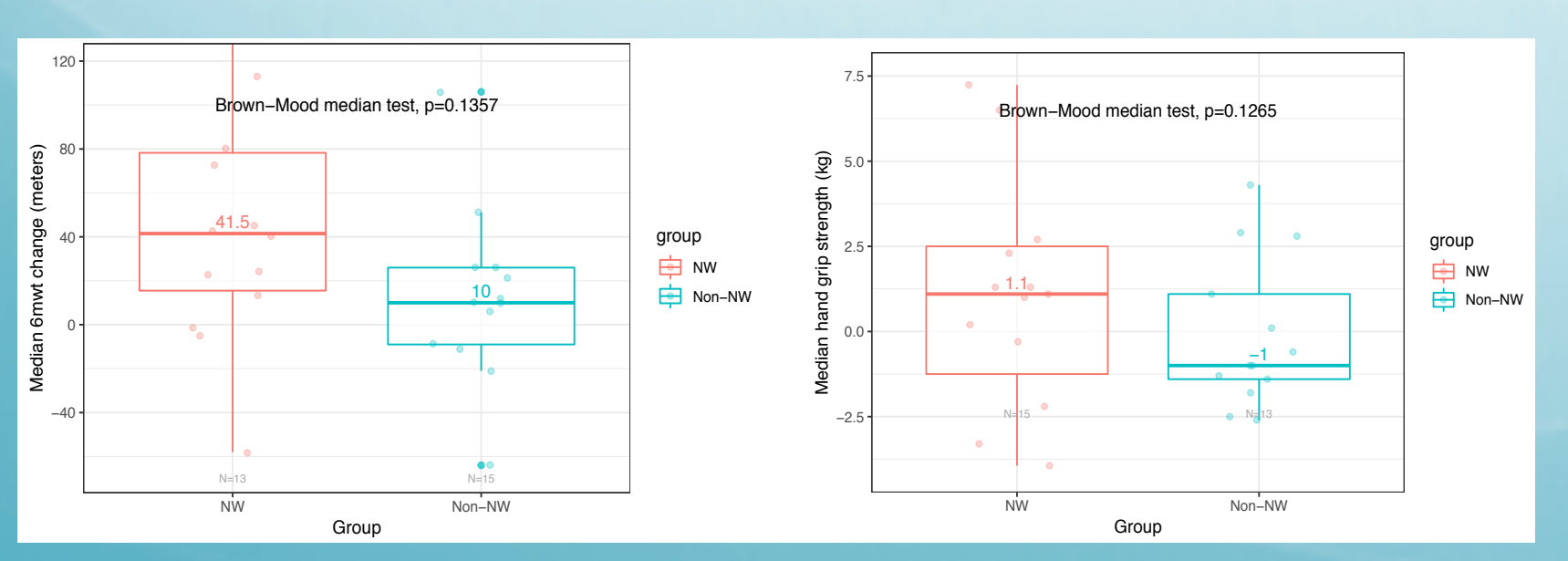

\section{Discussion/Implications}

A $41.5 \mathrm{~m}$ improvement in 6MWT achieved in the NW group exceeded the Minimal Clinically Important Difference of 14.0 $30.5 \mathrm{~m} \cdot{ }^{3}$ Although a greater number of participants is needed to confirm these findings more confidently, these encouraging results indicate that a group-based supervised Renal NW program may provide meaningful benefits to renal outpatients. The program is now adopted by the hospital.

\section{Dissemination plan/KT Approach}

Key messages were/are being shared via newsletters (i.e., allied health associations, Providence Health Care Communications internally \& externally), general and targeted social media (i.e., Urban Poling, BC Renal Agency, Kidney Foundation of Canada), and conference abstracts and presentations.

\section{Acknowledgements}

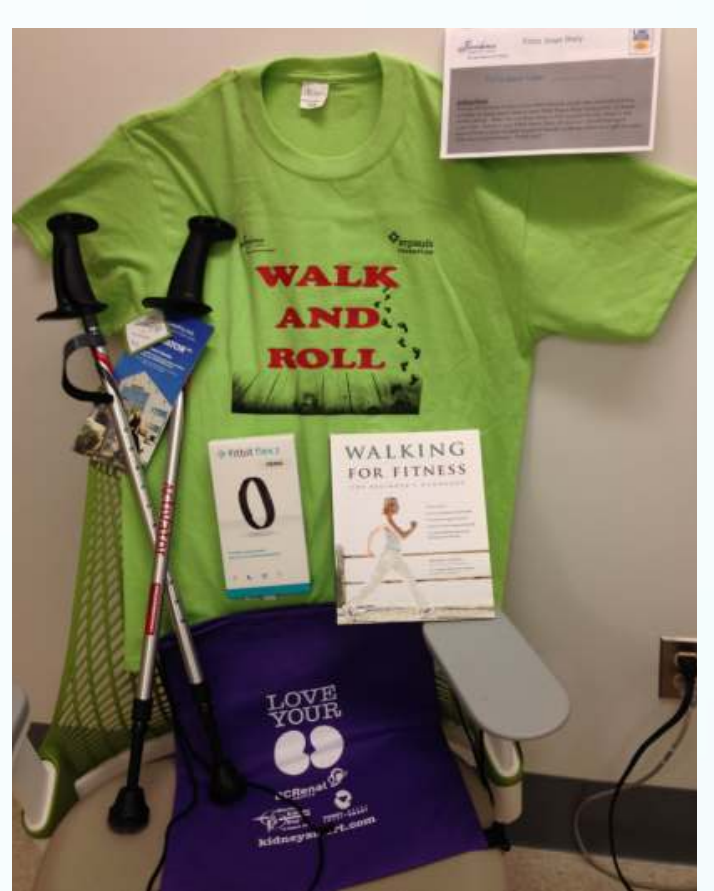

Nordic Walking Toolkit
The research team would like to thank the volunteers and participants in their study, especially their patient partner for his valuable feedback throughout the entire process from conception to T-shirt design and dissemination. They also express appreciation to the PHC Practice-based Research Challenge competition for funding the study, the St. Paul's Foundation for funding the Nordic walking toolkits, and renal leadership for their tangible and meaningful commitment to evidence-informed patient-centred care delivery by allocating funding to continue the Nordic walking program for patients.

\section{References}

1. Roshanravan B, Gamboa J, Wilund K. Exercise and CKD: Skeletal Muscle Dysfunction and Practical Application of Exercise to Prevent and Treat Physical Impairments in CKD. Am J Kidney Dis 2017 Jun;69(6):837-852.

2. Clarke AL, Young HM, Hull KL, Hudson N, Burton JO, Smith AC. Motivations and barriers to exercise in chronic kidney disease: a qualitative study. Nephrol Dial Transplant 2015 Nov;30(11):1885-1892.

3. Bohannon RW, Crouch R. Minimal clinically important difference for change in 6-minute walk test distance of adults with pathology: a systematic review. J Eval Clin Pract 2017 Apr;23(2):377-381. 